\title{
Intracranial radiotherapy with or without immune checkpoint inhibition for brain metastases: a systematic review and meta-analysis
}

\author{
Qian He, Chi Zhang, Sheng Tang, Jiayi Li, Qinglan Ren \\ Department of Oncology, The First Affiliated Hospital of Chongqing Medical University, Chongqing, China \\ Contributions: (I) Conception and design: All authors; (II) Administrative support: None; (III) Provision of study materials or patients: Q He, C \\ Zhang; (IV) Collection and assembly of data: Q He, S Tang, J Li; (V) Data analysis and interpretation: Q He, Q Ren; (VI) Manuscript writing: All \\ authors; (VII) Final approval of manuscript: All authors. \\ Correspondence to: Qinglan Ren. No. 1 Youyi Road, Yuanjiagang, Yuzhong District, Chongqing 400016, China. Email: renqlwu@163.com.
}

Background: With the development of immunotherapy in recent years, the prognosis of patients is expected to improve due to immune checkpoint inhibition (ICI) combined with radiotherapy (RT). However, studies on combination therapy (ICI + intracranial RT) have reported inconsistent results, and it is unclear whether the combination has increased toxicity. By analyzing the latest relevant literature, we performed a meta-analysis to further clarify the effectiveness and safety of intracranial RT combined with ICI in patients with brain metastases (BMs).

Methods: We searched PubMed, Embase and the Cochrane Library for published studies that compared the efficacy and safety of intracranial RT combined with ICI versus intracranial RT alone in the treatment of BMs. Overall survival (OS), local brain failure (LBF), distant brain failure (DBF), and radiation necrosis (RN) were pooled with the use of the hazard ratio (HR) or odds ratio (OR).

Results: A total of 26 retrospective observation cohort studies were included, and over 1,500 patients who received ICI and intracranial RT were evaluated. Compared with intracranial RT alone, RT combined with ICI significantly improved OS in patients with $\mathrm{BMs}$ [HR $=0.55,95 \%$ confidence interval (CI): 0.48-0.64, $\mathrm{P}<0.001$ when OS was defined from the date of diagnosis of $\mathrm{BMs}$; $\mathrm{HR}=0.45,95 \%$ CI: 0.39-0.52, $\mathrm{P}<0.001$ when OS was defined from the date of RT], though the risk of RN was similar to that of RT alone (HR $=1.27,95 \%$ CI: 0.58-2.79, P=0.55). However, significant improvement in LBF and DBF was not obtained with RT combined with ICI (1-year LBF: OR =1.71, 95\% CI: 0.38-7.67, P=0.48; LBF: HR =0.49, 95\% CI: $0.28-0.87, \mathrm{P}=0.01$; 1-year DBF: OR =1.05, 95\% CI: 0.47-2.33, P=0.90).

Conclusions: ICI combined with intracranial RT confers a significant OS benefit for patients with BMs without significantly increasing treatment-related toxicity, but further research regarding the specific details of combined treatment application is needed to improve the survival and quality of life of patients with BMs.

Keywords: Immune checkpoint inhibition (ICI); intracranial radiotherapy; radiation therapy; stereotactic radiosurgery (SRS); brain metastases (BMs)

Submitted Feb 07, 2020. Accepted for publication Sep 02, 2020.

doi: 10.21037/tcr-20-902

View this article at: http://dx.doi.org/10.21037/tcr-20-902

\section{Introduction}

Brain metastasis (BM) is the most common type of intracranial tumor and mainly originates from melanoma and lung cancer. As patients with BM have a short survival time, improving the treatment effect for BMs has gradually become a major focus of research. At present, immunotherapy shows good therapeutic prospects for malignant tumors. However, its role in BMs has been 
overlooked, as it is generally believed that antitumor drugs exert minimal efficacy through the blood-brain barrier (BBB). Nonetheless, recent data have shown that the BBB can be destroyed by BMs, tumor-infiltrating lymphocytes (TILs) are significant in BMs, and the brain is no longer a strict "immune privilege" environment (1). Indeed, a certain level of BBB permeability of immune checkpoint inhibition (ICI) has been reported in BM (2). Clinical trials (CheckMate 017 and CheckMate 057) have also indicated that some patients with BMs experience improved overall survival (OS) after using nivolumab (3). Furthermore, an increasing number of studies have reported a certain response rate for intracranial tumors with various immune checkpoint inhibitors, possibly enhancing the survival time of patients $(1,4-6)$.

Studies have revealed that radiotherapy (RT), as a mainstay in the treatment of BMs, is able to promote antitumor immune effects by inducing immunogenic cell death, exposing tumor-associated antigens, activating dendritic cells, reprogramming the tumor microenvironment, and enhancing expression of intercellular adhesion molecule-1 (ICAM-1), Fas and major histocompatibility complex I (MHCI) $(7,8)$. Additionally, based on preclinical models, high-dose fractionated RT is immunogenic, potentially opening the BBB to facilitate the entry of immune checkpoint inhibitors and TILs into BMs (9). Moreover, some studies have found that RT can upregulate expression of PD-L1 and suppress antitumor immune effects (10). Interestingly, both the immune-enhancing effects and upregulation of PD-L1 expression after intracranial RT can theoretically increase the efficacy of immunotherapy. Therefore, RT combined with ICI may offer favorable benefits to patients with BMs.

Accordingly, the synergistic antitumor effect of RT and ICI in BMs is gradually being investigated. To date, quite a few studies have demonstrated that combination treatment is effective (11), though some have shown that combination therapy only improves the tumor regression rate and has no obvious effect on OS (12). Indeed, a small number of studies report that combined treatment has no significant advantage on either OS or the local control of intracranial BMs (13-16). In this systematic review and meta-analysis (SRMA), we aimed to further evaluate the efficacy and safety of ICI and intracranial RT combination therapy. We present the following article in accordance with the PRISMA reporting checklist (available at http:// dx.doi.org/10.21037/tcr-20-902).

\section{Methods}

\section{Search strategy and selection criteria}

We conducted a literature search in PubMed, Embase and the Cochrane Library from inception to November 2019. We used "immune checkpoint inhibition", "immunotherapy", "intracranial radiotherapy", "radiation", "stereotactic radiosurgery", and "brain metastases" as key words for our search.

The inclusion criteria of our search were designed around the "PICOS" principle, as follows: Population, patients with BMs; Intervention, ICI with intracranial RT; Comparison, intracranial RT alone; Outcome, the hazard ratio (HR) of OS, local brain failure (LBF) or radiation necrosis $(\mathrm{RN})$; and Study, randomized trial or cohort study. All articles were independently reviewed by two investigators.

Exclusion criteria for the included articles were as follows: (I) repeated publication of data; (II) not a comparative study; (III) not published in English or Chinese; (IV) not treating BMs or including extracranial radiation; (V) not ICI combined with RT vs. RT alone; and (VI) not reported HRs for OS, LBF or RN.

\section{Data extraction and quality assessment}

Two investigators independently extracted the following data from each study: the publication year, number of institutions, type of study design, study period, number and median age of enrolled patients, median follow-up time, primary carcinoma of BM, radiation type, regimens of ICI, and outcomes of the various groups. Outcomes were extracted as follows: HRs for OS, LBF or RN and the incidence of 1-year LBF or 1-year DBF. When disagreements occurred, discussions were made with a third author to reach a consensus.

The quality of the studies (e.g., selection, comparability, and outcome) included was assessed using the NewcastleOttawa Scale (NOS) by two independent investigators. The total score was 9 , with $1-5$ being of low quality and 6-9 of high quality. Disagreements were discussed with a third author to reach a consensus.

\section{Data analyses and statistical methods}

Meta-analyses were conducted with RevMan 5.3 software (The Nordic Cochrane Center, Cochrane Collaboration). HRs were used to evaluate OS (primary endpoint), LBF 

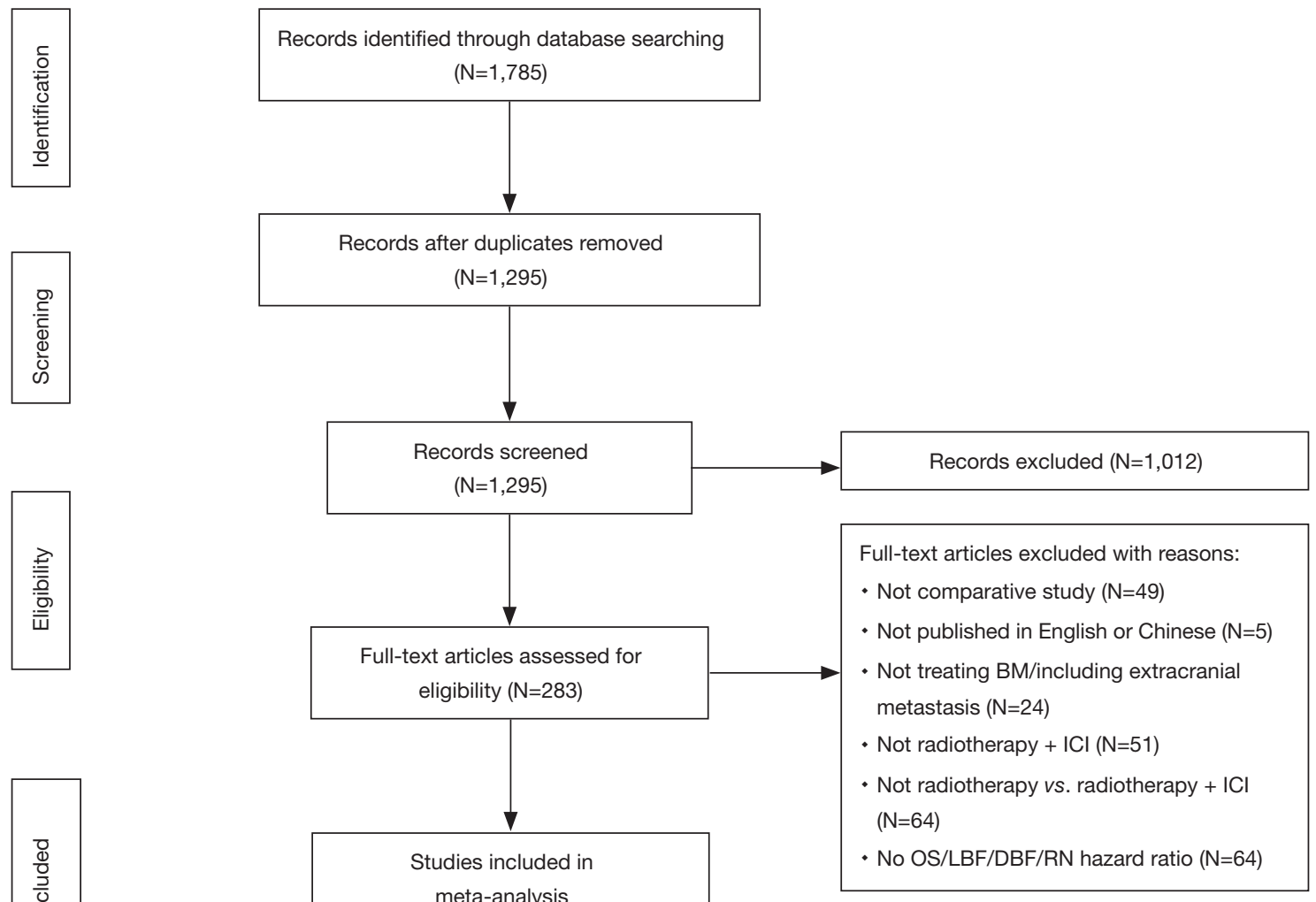

$(\mathrm{N}=26)$

Figure 1 The flow diagram described our process of selection studies. We finally included 26 retrospective cohort studies involving approximately 1,500 patients who received a combination of ICI and intracranial RT. BM, brain metastases; DBF, distant brain failure; ICI, immune checkpoint inhibition; LBF, local brain failure; N, number; RN, radiation necrosis.

and RN. Subgroup analyses were applied to assess the results for patients with different RT forms and primary tumor types. The incidence of 1-year LBF and 1-year $\mathrm{DBF}$ was evaluated through the odds ratio (OR). All data were extracted from the studies, and the $95 \%$ CI was collected. When $\mathrm{I}^{2}<33$, the fixed-effects (FE) model was employed to pool the outcomes of the studies included in the meta-analysis; when $\mathrm{I}^{2} \geq 33$, the random-effects (RE) model was applied. The outcomes are shown as forest plots, which also represent the heterogeneity statistics. Heterogeneity was estimated through the chi-squared test and $\mathrm{I}^{2}$ statistics, whereby the larger $\mathrm{I}^{2}$ was, the greater was the heterogeneity; $\mathrm{I}^{2}=0 \%$ was considered no heterogeneity, and $\mathrm{I}^{2}>50 \%$ was considered high heterogeneity. Significance was set at $\mathrm{P}<0.05$. A funnel plot was used to assess publication biases for primary outcomes. Each study was removed sequentially for sensitivity analysis. If bias was suspected, the pooled effect size was recalculated with the trim-and-fill method.

\section{Results}

\section{Included studies and study quality}

A total of 1,785 relevant studies were retrieved from databases, and 1,295 articles remained after eliminating duplicate studies using literature management software. We removed 1,012 papers by abstract screening and then assessed the full texts of the remaining papers using the selection criteria. Ultimately, 26 retrospective observational cohort studies were included (Figure 1), and over 1,500 patients who received combination treatment of ICI and intracranial RT were assessed. Table 1 summarizes the main baseline characteristics and survival outcomes. The cohort studies that we included were generally of high quality, with NOS scores greater than 6 (Table S1).

\section{OS}

The HR of OS was calculated in 22 studies, and OS 


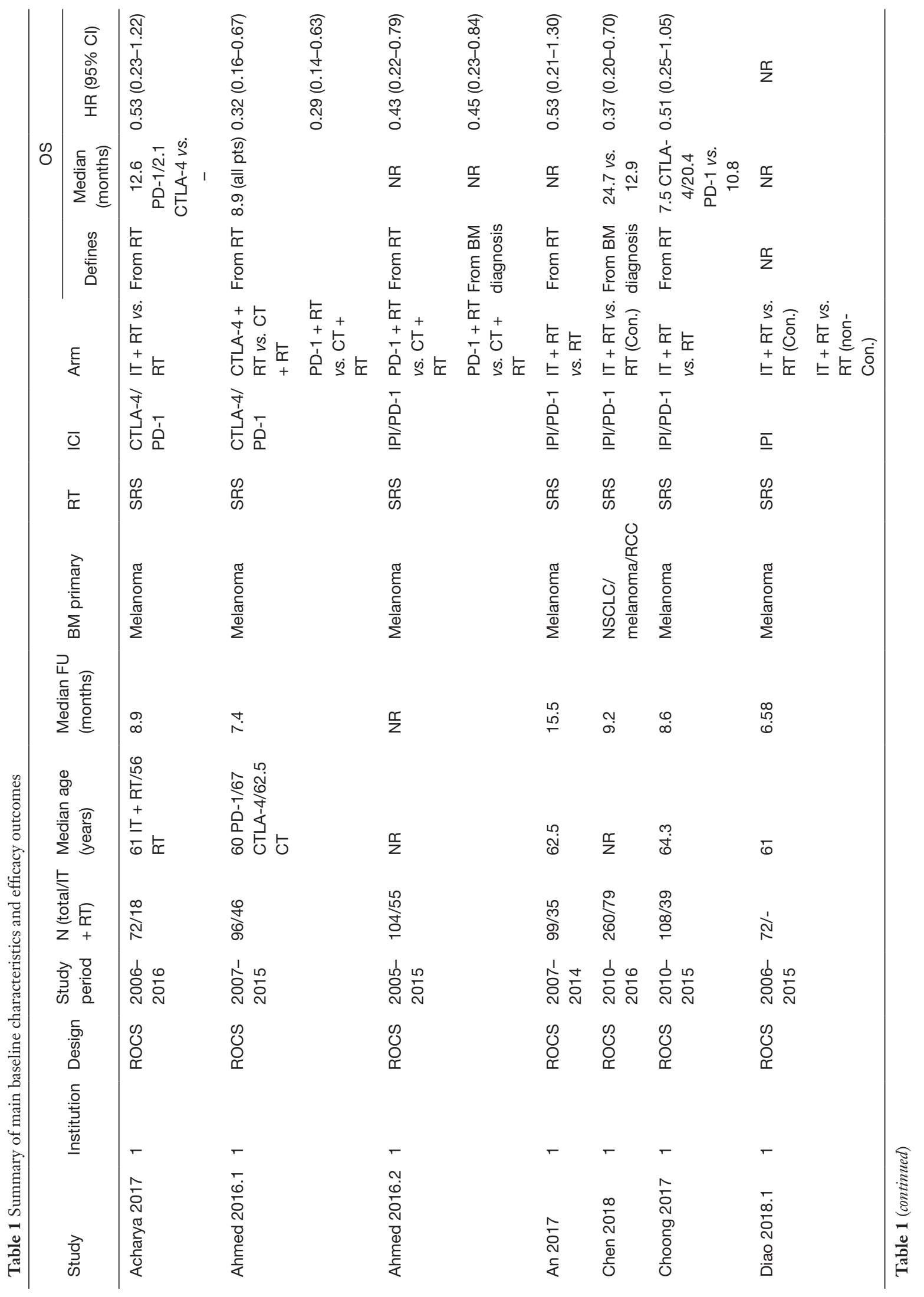




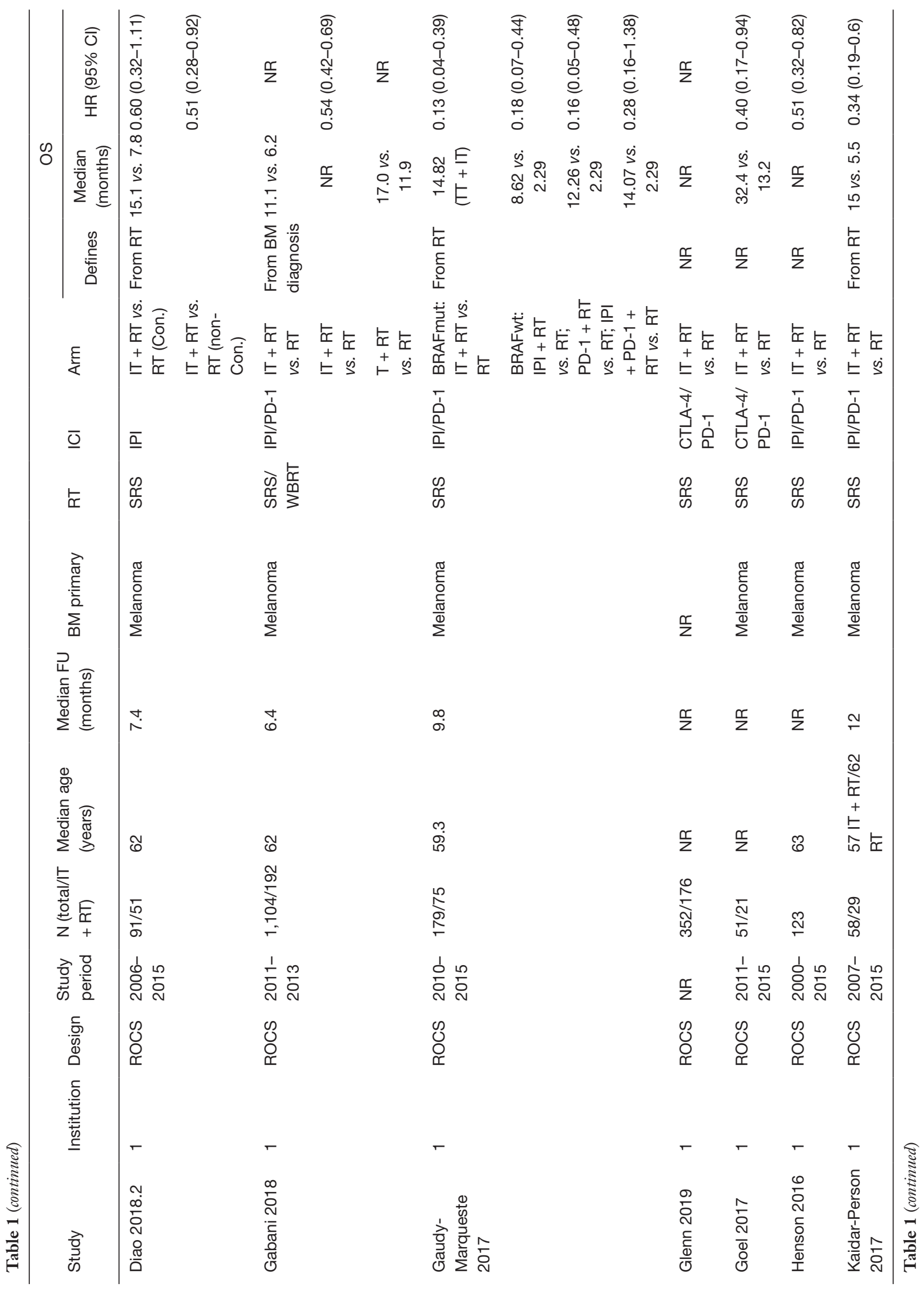




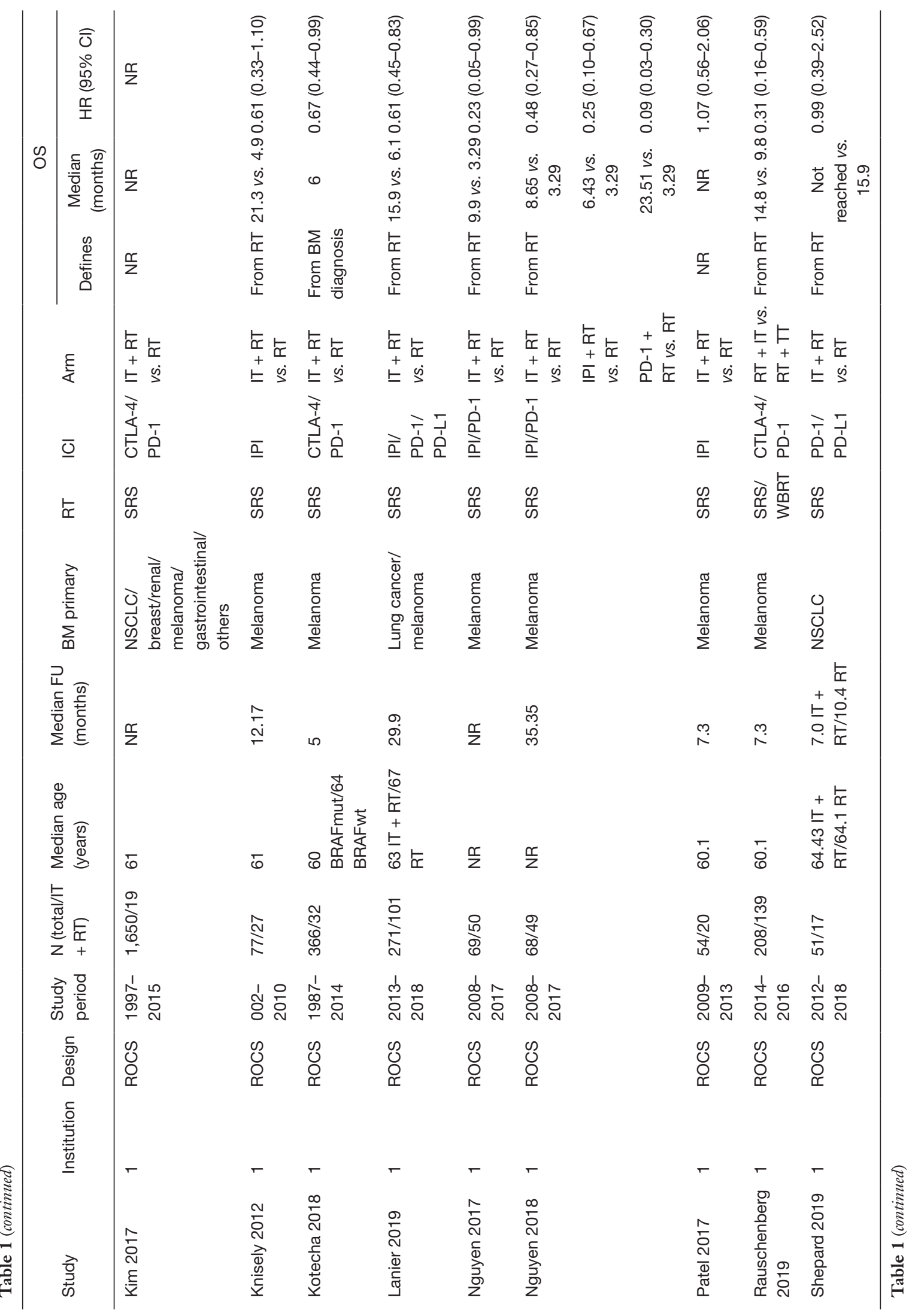




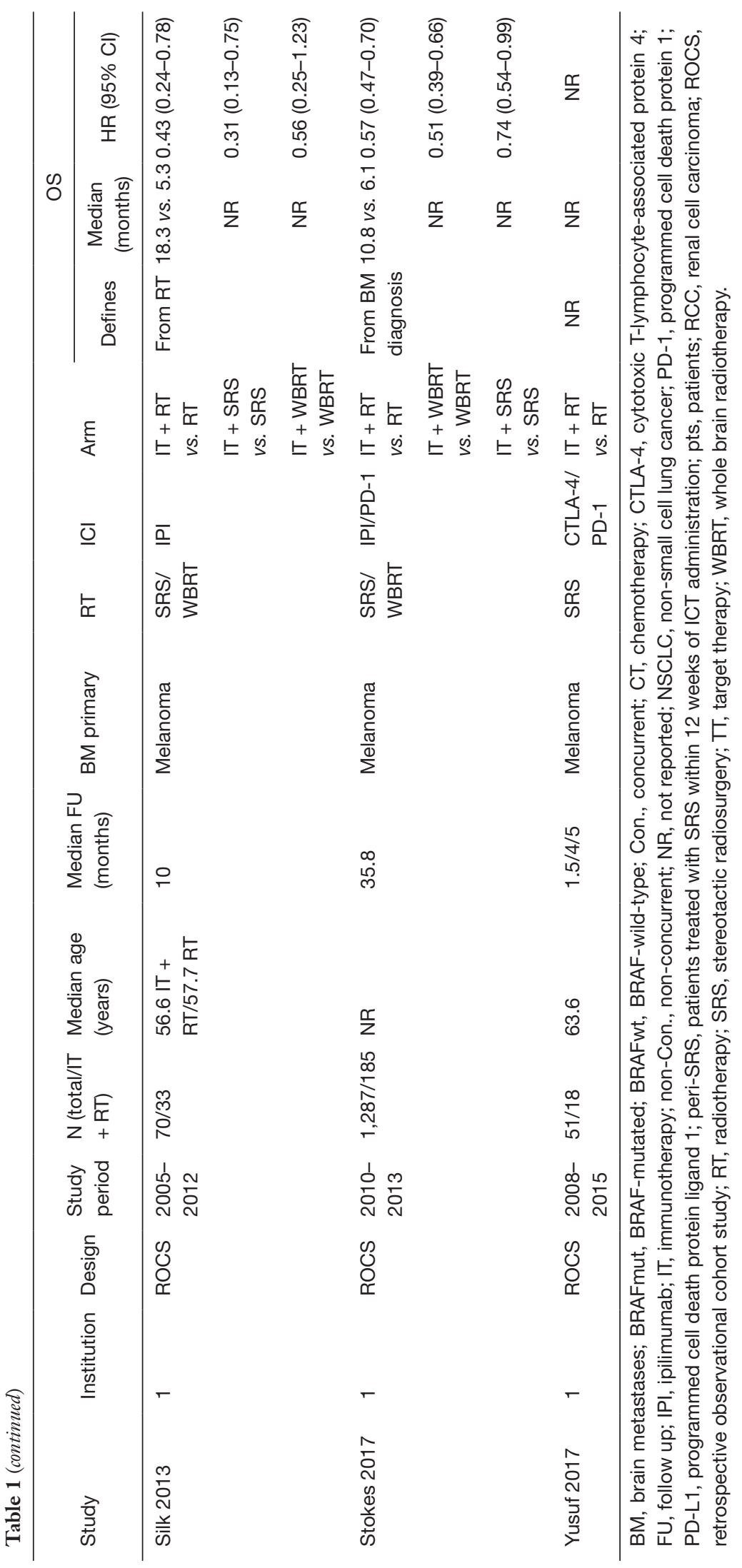


was defined from the date of diagnosis of BMs $(\mathrm{n}=5)$ (17-21) or the date of intracranial RT ( $\mathrm{n}=15$ studies) $(12,18,22-34)$. As statistical heterogeneity among the study outcomes was not high $\left(\mathrm{I}^{2}=0 \% ; \mathrm{I}^{2}=29 \%\right.$, respectively), the FE model was applied. The pooled HR demonstrated that the use of ICI significantly lowered the risk of death, regardless of whether OS was defined from the date of diagnosis of BMs or the date of RT (HR $=0.55$, 95\% CI: 0.48-0.64, P<0.001; $\mathrm{HR}=0.45$, 95\% CI: $0.39-0.52, \mathrm{P}<0.001$, respectively) (Figure 2). OS was not clearly defined in three studies [Goel 2017 (35), HR =0.40, 95\% CI: 0.17-0.94; Henson 2016 (36), HR =0.51, 95\% CI: 0.32-0.82; Patel 2017 (37), HR $=1.07$, 95\% CI: 0.56-2.06]; therefore, these studies were not included in the pooled HR. Through subgroup analysis (Figures 2,3), we also found that ICI significantly reduced the risk of death when combined with stereotactic radiosurgery (SRS), and the pooled HR of OS in the SRS subgroup was 0.46 (95\% CI: $0.39-0.53, \mathrm{P}<0.001, \mathrm{I}^{2}=31 \%$ ) and 0.59 (95\% CI: $0.44-0.80, \mathrm{P}<0.001, \mathrm{I}^{2}=41 \%$, RE model, Figure 3) when OS was defined from the date of RT ( $\mathrm{n}=14$ studies) and the diagnosis of $\mathrm{BMs}$ ( $\mathrm{n}=4$ studies), respectively. When ICI was combined with whole-brain radiotherapy (WBRT), there was no obvious difference in survival when OS was defined from the date of RT ( $\mathrm{n}=1$ study, HR $=0.56$, 95\% CI: $0.25-1.23, \mathrm{P}=0.15$ ). When OS was defined from the date of diagnosis of BMs, combination therapy appeared to have a survival advantage in the WBRT subgroup $(\mathrm{n}=2$ studies, HR $=0.53,95 \%$ CI: $0.44-0.63, \mathrm{P}<0.001, \mathrm{I}^{2}=0 \%$ ). Additionally, compared to RT alone, the OS benefit (defined from the date of RT) of combination therapy was significant in both the melanoma subgroup ( $\mathrm{n}=13$ studies) and in the other cancer subgroup (lung cancer and/or melanoma and/ or renal cell carcinoma, $\mathrm{n}=2$ studies), with pooled HRs of 0.40 and 0.64 , respectively (95\% CI: $0.34-0.47, \mathrm{P}<0.001$, $\mathrm{I}^{2}=8 \%$; $95 \%$ CI: $0.48-0.86, \mathrm{P}=0.003, \mathrm{I}^{2}=0 \%$, respectively).

\section{$L B F$}

LBF was defined as the date of first tumor progression in the radiation field, and LBF was evaluated in 7 studies. Three of the studies calculated the incidence of 1 -year LBF for 420 patients $(26,28,30)$, and heterogeneity between the results was high $\left(\mathrm{I}^{2}=84 \%\right)$. RE model analysis revealed no significant difference in 1-year LBF between the two groups $(\mathrm{OR}=1.71,95 \% \mathrm{CI}: 0.38-7.67, \mathrm{P}=0.48)$ (Figure 4). Interestingly, four other studies with some heterogeneity $\left(\mathrm{I}^{2}=51 \%\right)$ calculated the HR of $\operatorname{LBF}(17,22,38,39)$ and found that combination therapy may be a favorable predictor of LBF risk reduction $(\mathrm{HR}=0.49,95 \%$ CI: $0.28-0.87, \mathrm{P}=0.01$, RE model) (Figure 5).

\section{Distant brain failure (DBF)}

DBF was defined as the emergence of a new BM or tumor progression outside the prior radiation treatment field in the brain. Seven articles ( $\mathrm{n}=671$ patients) assessed DBF $(12,18,23,26,28,30,37)$. An RE model was used for the meta-analysis due to high heterogeneity $\left(\mathrm{I}^{2}=73 \%\right)$, and the outcomes suggested no significant advantage in 1-year DBF between patients who received combination therapy and those who received $\mathrm{RT}$ alone ( $\mathrm{OR}=1.05,95 \% \mathrm{CI}: 0.47$ 2.33, $\mathrm{P}=0.90$ ) (Figure 6).

\section{Toxicity}

$\mathrm{RN}$ was defined based on pathological and/or radiographic evidence, and enhanced circular lesions usually indicate RN. Short-term follow-up using an institutional algorithm, positron emission tomography (PET), cerebral blood volume MRI, or surgical assessment (biopsy or resection) is essential for distinguishing tumor recurrence or progression from RN. Five studies analyzed RN, three of which included the HR $(17,40,41)$. The RE model was used because of high heterogeneity $\left(\mathrm{I}^{2}=49 \%\right)$, and the pooled $\mathrm{HR}$ of $\mathrm{RN}$ showed no significant difference in $\mathrm{RN}$ between the two groups ( $\mathrm{HR}=1.27,95 \% \mathrm{CI}$ : 0.58-2.79, $\mathrm{P}=0.55$ ), revealing that combination therapy did not increase the risk of RN (Figure 7).

\section{Publication bias and sensitivity analysis}

A funnel plot was constructed for OS (defined from the date of RT). The funnel plots were basically symmetrical, suggesting no significant publication bias (Figure 8). Sensitivity analysis was performed by removing each study sequentially, with consistent outcomes.

\section{Discussion}

Although some studies have shown that ICI combined with RT can bring clinical benefits to patients with BMs and that the toxicity is acceptable, no large-scale randomized controlled trial (RCT) has yet to confirm this finding. Therefore, the clinical effect and toxicity of combined therapy need to be further examined to improve the level 

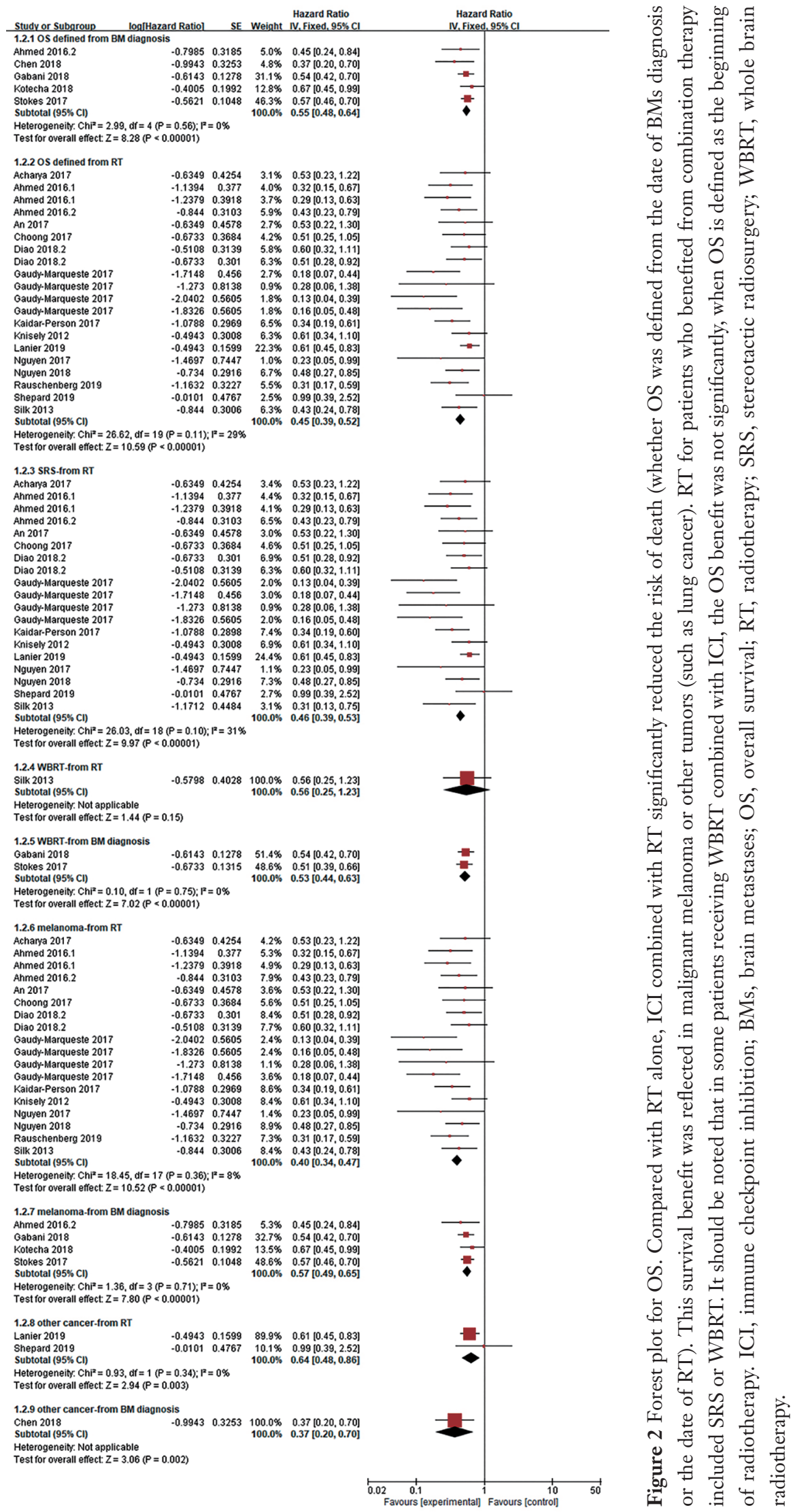


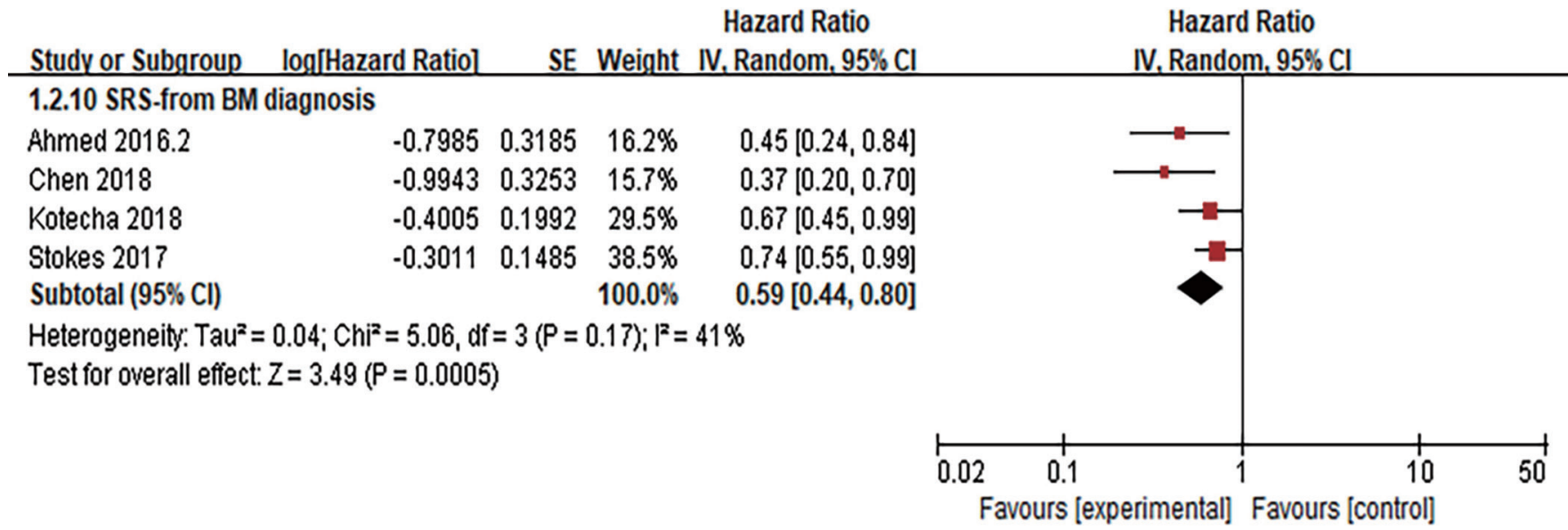

Figure 3 Forest plot for OS. SRS combined with ICI also showed OS advantages compared with SRS alone, when OS is defined as starting from the diagnosis of BMs. ICI, immune checkpoint inhibition; SRS, stereotactic radiosurgery; BMs, brain metastases.

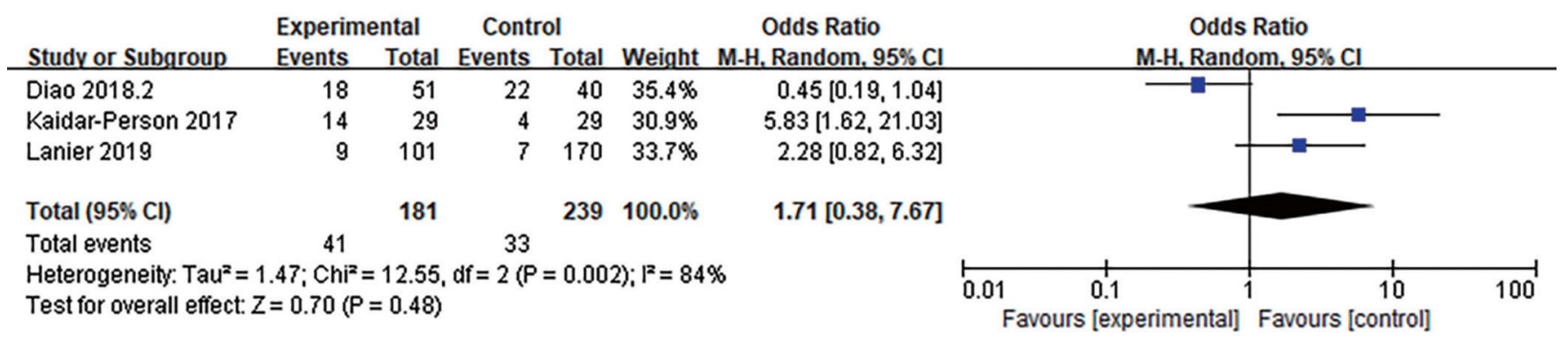

Figure 4 Forest plot for 1-year LBF. There was no significant difference in 1-year LBF between the ICI combined with RT group and RT alone group. ICI, immune checkpoint inhibition; RT, radiotherapy; LBF, local brain failure.

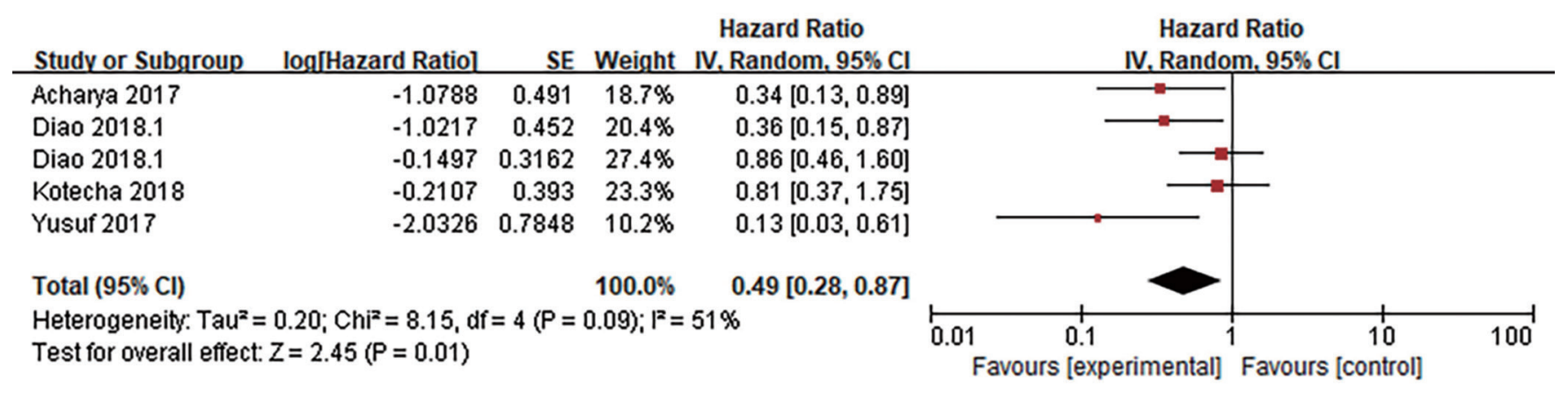

Figure 5 Forest plot for the pooled HR of LBF. The combination of ICI and RT may be a favorable predictor of LBF risk reduction. HR, hazard ratio; LBF, local brain failure; ICI, immune checkpoint inhibition; RT, radiotherapy. 


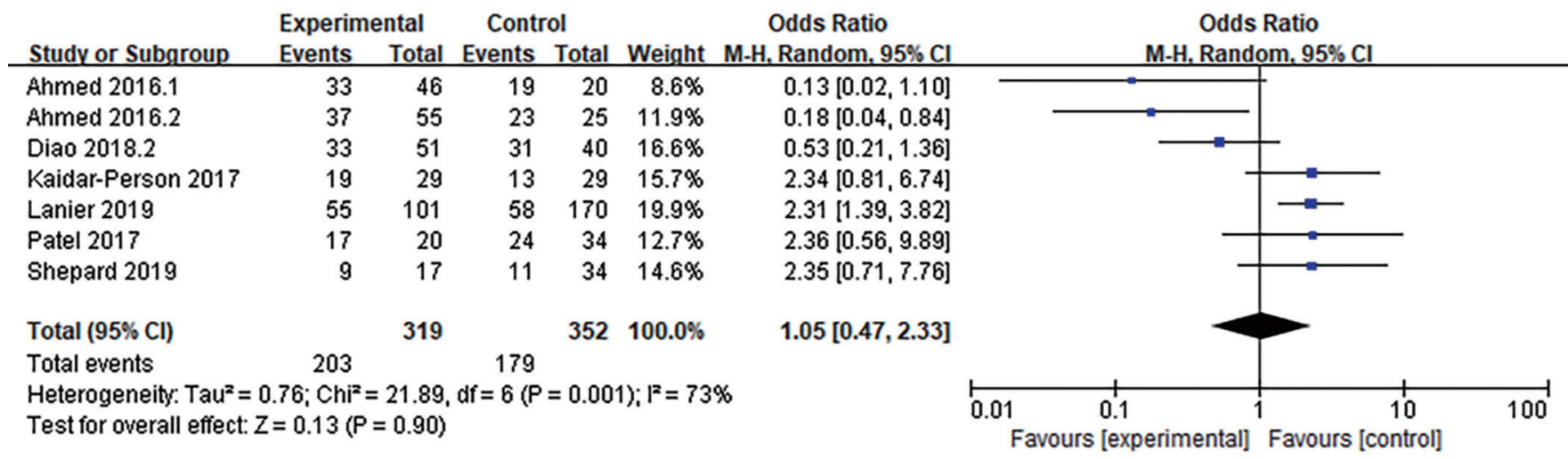

Figure 6 Forest plot for 1-year DBF. There was no significant advantage in 1-year DBF between patients who received combination therapy and those who received RT alone. DBF, distant brain failure; RT, radiotherapy.

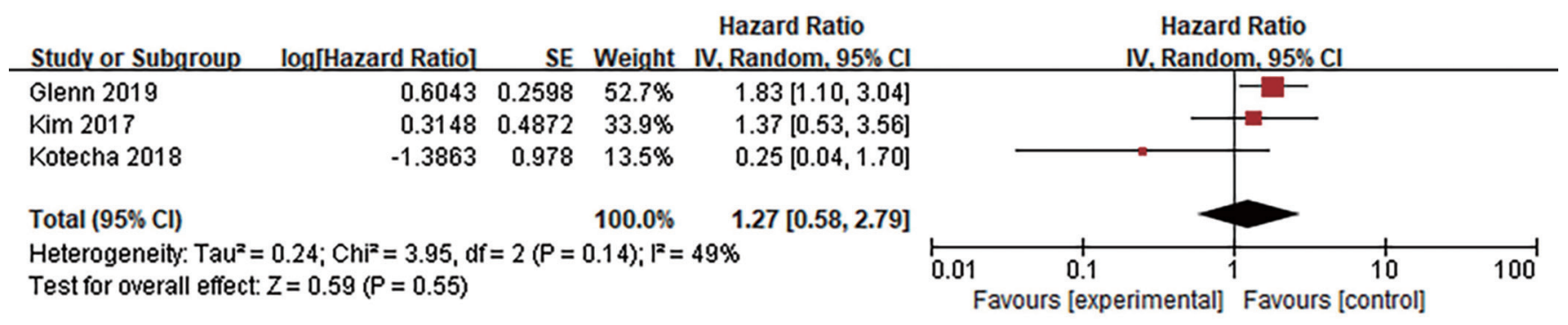

Figure 7 Forest plot for the pooled HR of RN. ICI combined with RT did not increase the risk of RN. HR, hazard ratio; ICI, immune checkpoint inhibition; RT, radiotherapy; RN, radiation necrosis.

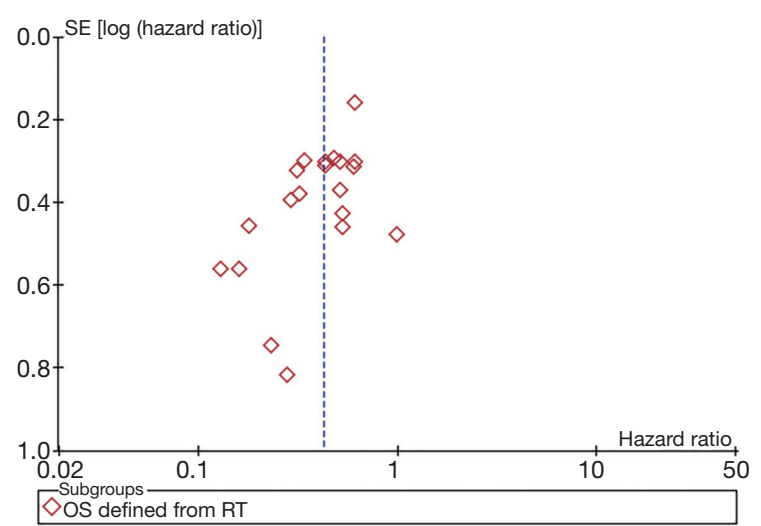

Figure 8 Funnel plot for OS defined from the date of RT. There was no significant publication bias. OS, overall survival; RT, radiotherapy.

of evidence and guide clinical practice. A previous metaanalysis that included studies before April 2018 evaluated the efficacy and safety of combination therapy for
BMs (42), in which the primary endpoints were the median OS and 1- and 2-year OS rates. Because ICI combined with RT for BMs has become a focus and outcomes of related studies have been released successively, we designed and implemented this meta-analysis to include the latest research outcomes. The primary endpoint of this metaanalysis was the pooled HR for OS, which is highly capable of assessing survival benefits; the toxicity of combined therapy was assessed at the same time. This meta-analysis provides the latest data on the safety of treatment and its impact on patients' quality of life.

This meta-analysis indicated that in terms of OS benefits, combination therapy conferred a significant OS benefit compared with RT alone, regardless of whether OS was defined from the date of $\mathrm{BM}$ diagnosis or the date of RT $(\mathrm{HR}=0.55, \mathrm{P}<0.001 ; \mathrm{HR}=0.45, \mathrm{P}<0.001$, respectively), especially when combined with $\mathrm{SRS}(\mathrm{HR}=0.46, \mathrm{P}<0.001)$. No statistically significant difference in the OR of 1-year LBF $(\mathrm{OR}=1.71, \mathrm{P}=0.48)$ or 1 -year DBF $(\mathrm{OR}=1.05$, 
$\mathrm{P}=0.90$ ) was found between the two groups, which suggests no corresponding significant trends in LBF or DBF. However, when the pooled HR was calculated to assess LBF, the combination therapy appeared to achieve better local control $(\mathrm{HR}=0.49, \mathrm{P}=0.01$ ). Although no significant improvement in intracranial control was found, there was still a survival advantage in the ICI group, indicating that these survival benefits may result from improvements in systemic disease control. This was consistent with the finding that the main limiting survival factor for patients with $\mathrm{BMs}$ is systemic disease rather than intracranial disease $(26,43)$. Overall, there is no consensus to date on whether combination therapy will have better intracranial control. Some studies have reported no significant differences in LBF or DBF $(37,44,45)$, whereas others have revealed that the appropriate timing of combination therapy might improve LBF or DBF $(24,38,46)$. Nevertheless, most of these studies were retrospective and showed interstudy heterogeneity. Thus, further prospective studies are needed to further investigate intracranial control. Moreover, this meta-analysis detected no significant difference in $\mathrm{RN}$ risk between the two groups, indicating that combination therapy is generally safe and acceptable, consistent with previous studies (47).

When we analyzed the research, we found that the level of PD-L1 expression in tumors, which is closely related to the efficacy of immunotherapy, appeared to have no significant correlation with the efficacy of combination therapy. This may be partly due to the apparent heterogeneity of PD-L1 in tumor tissues and the influence of specific antibodies used in immunohistochemistry (12). Notably, PD-L1 expression levels may change significantly during treatment. For example, animal trials have shown that PD-L1 expression increases after RT (48). Furthermore, clinical trials (e.g., the PEMBRO-RT study) comparing the efficacy of pembrolizumab with the combination of pembrolizumab and stereotactic body radiotherapy (SBRT) found that in the combination treatment group, PD-L1-negative advanced lung cancer patients had greater benefits than did PD-L1positive patients (49). Therefore, it is highly possible that more patients may benefit from the combination of ICI and RT.

According to this meta-analysis, combination therapy was used mainly in patients with melanoma and non-small cell lung cancer (NSCLC), the treatment of which was associated with the mutation status of key driver genes. There is also evidence that BRAF mutation (BRAFmut) is a positive prognostic factor for melanoma (i.e., prolonged
OS) when patients receive ICI and intracranial RT (33). Gaudy-Marqueste $e t$ al. studied the effect of ICI and/or targeted therapy (TT) on the survival of 179 patients with melanoma BMs who received intracranial SRS and found that BRAF wild-type (BRAFwt) patients who received antiPD-1 or BRAFmut patients who received anti-PD-1 and BRAF inhibition (BRAFi) therapy had the best OS (12.26 and 14.82 months, respectively) (27). Furthermore, the multivariate analysis of Kotecha et al. (17) showed BRAFi and anti-PD-1/CTLA-4 therapies to be significantly associated with OS and BRAFmut status to be a predictor of a low risk of $\mathrm{RN}$ (HR $=0.27,95 \% \mathrm{CI}$ : 0.07-0.84). Notably, outcomes in their study may have been affected by imbalanced baseline data. Additionally, Cinausero et al. found that in nonsquamous NSCLC, patients with KRAS mutation (KRASmut) had a better response to PD-1 inhibition than did patients with KRAS wild-type (KRASwt), with prolonged progression-free survival (PFS) and OS. These authors considered KRAS mutations and deletions in ERBB family genes (including EGFR, ERBB2 and ERBB4) to be favorable predictors for PD-1 inhibition (50). These outcomes suggest that the detection of tumor-specific driver genes in different tumors during the combination of ICI and RT may help predict the efficacy of such combined therapy. We will further focus on related research in this area.

Although combination therapy may improve the OS of patients, different disease-specific grading prognostic factors (DS-GPAs) may lead to different efficacies of combination therapy. The latest versions of melanoma DS-GPA (melanoma-molGPA) (51) and lung cancer DSGPA (lung-molGPA) (52) share four important survival prognostic factors: age, Karnofsky performance status (KPS) score, extracranial metastases and number of BMs. Additionally, melanoma-molGPA includes BRAF status, and lung-molGPA includes EGFR and ALK statuses. Multivariate analysis has shown that higher DS-GPA scores, such as young age, no extracranial metastases, high KPS scores and few BMs, are significantly related to improved OS $(17,27)$. Moreover, retrospective studies have found that patients with a KPS $<90$ (9) or $<80$ (Singh et al. 2019) or patients with lung-mol GPA score $<1.5$ (13) often have poor OS.

By analyzing the extracted literature in detail, we found that when ICI was combined with RT, the specific strategy of RT used (such as selection of the RT form, irradiation field, and timing and sequence of the combination therapy) also affected efficacy. Regarding the RT form, there 
were limited data available for WBRT, and no consensus concerning whether WBRT combined with ICI could achieve similar survival benefits was reached $(20,21,33,34)$. Regardless, this meta-analysis did reveal that SRS combined with ICI conferred a marked survival advantage for patients ( $\mathrm{HR}=0.46, \mathrm{P}<0.001 ; \mathrm{HR}=0.59, \mathrm{P}<0.001$, when $\mathrm{OS}$ was defined from the date of RT or the date of diagnosis of BMs, respectively). The synergistic effect between RT and immunotherapy depends on the activation of immune cells (such as T lymphocytes), which are easily killed by RT. Moreover, RT kills not only TILs but also peripheral blood lymphocytes passing through the irradiation field and may induce lymphopenia. Of note, there are emerging data revealing that severe lymphopenia is associated with a poor prognosis in patients with different tumors (53-55). Therefore, the size of the radiation field should be reasonably limited when investigating combination therapy (7). We also need to pay attention to the timing of combination therapy. For instance, a preclinical study by Dovedi et al. using mouse models (56) indicated that the PD-L1 expression level gradually increased after RT, reached a peak after 72 hours, and gradually decreased after 7 days. Their study also showed that compared with the RT-only group, ICI on the 1st or 5th day of the RT cycle improved OS but that no advantage was obtained with sequential treatment with ICI 7 days after RT completion. Subsequent analysis of the PACIFIC study also revealed that patients who received ICI within 14 days after the last RT before randomization benefited more than those who received ICI later (48). Additionally, many retrospective analyses suggest that concurrent combination therapy can better improve the prognosis of patients with BMs than can nonconcurrent therapy $(57,58)$. Regarding the combination sequence, some scholars retrospectively analyzed 139 melanoma patients with BMs who received ICI within 6 weeks of RT and found no significant difference in the median survival time, regardless of whether ICI was used before, during or after RT ( $\mathrm{P}=0.72)$ (33). However, more data are needed to clarify the timing and sequence of combination therapy. Nonetheless, studies on the toxicity and side effects of immunotherapy combined with RT report that neither the timing nor sequence of combination therapy significantly increase the risk of toxicity (47).

\section{Strengths and limitations}

The main limitations of our study are as follows: (I) The studies included in this meta-analysis are all retrospective, and the results were limited by the preference of institutional treatment options. In some studies, the number of patients was small, patients were poorly matched, baseline data were incomplete, the timing of combination therapy differed, and the primary tumor types included were not sufficiently comprehensive. Overall, our analysis and findings are hypothesis generating, and large prospective randomized clinical trials will further validate the efficacy and safety of combination therapy. (II) The studies included a long-time span. The treatment strategy of ICI has gradually changed with its development, which may have affected the results. (III) The populations involved were not comprehensive, and whether different populations have similar effects needs further research. (IV) There were insufficient data for in-depth analyses of OS, LBF and DBF, and more clinical data are needed to screen the best beneficiaries and support the effect of combination therapy on intracranial control. (V) In our study, combination therapy toxicity was evaluated mainly through $\mathrm{RN}$, and more data are needed to evaluate intratumoral bleeding and other acute or chronic toxicities.

\section{Conclusions}

In our meta-analysis, the combination of ICI and intracranial RT for patients with BMs was associated with prolonged OS, and toxicity was tolerable. However, the efficacy of intracranial control needs further study. In the era of immunotherapy, larger prospective randomized trials are needed to further explore the strategy of combination therapy and optimize the combination plan to prolong the survival of patients and improve their quality of life.

\section{Acknowledgments}

Funding: None.

\section{Footnote}

Reporting Checklist: The authors have completed the PRISMA reporting checklist. Available at http://dx.doi. org/10.21037/tcr-20-902

Conflicts of Interest: All authors have completed the ICMJE uniform disclosure form (available at http://dx.doi. org/10.21037/tcr-20-902). The authors have no conflicts of 
interest to declare.

Ethical Statement: The authors are accountable for all aspects of the work in ensuring that questions related to the accuracy or integrity of any part of the work are appropriately investigated and resolved.

Open Access Statement: This is an Open Access article distributed in accordance with the Creative Commons Attribution-NonCommercial-NoDerivs 4.0 International License (CC BY-NC-ND 4.0), which permits the noncommercial replication and distribution of the article with the strict proviso that no changes or edits are made and the original work is properly cited (including links to both the formal publication through the relevant DOI and the license). See: https://creativecommons.org/licenses/by-nc$\mathrm{nd} / 4.0 /$.

\section{References}

1. Kamath SD, Kumthekar PU. Immune Checkpoint Inhibitors for the Treatment of Central Nervous System (CNS) Metastatic Disease. Front Oncol 2018;8:414.

2. Cohen JV, Kluger HM. Systemic Immunotherapy for the Treatment of Brain Metastases. Front Oncol 2016;6:49.

3. Brahmer J, Reckamp KL, Baas P, et al. Nivolumab versus Docetaxel in Advanced Squamous-Cell Non-Small-Cell Lung Cancer. N Engl J Med 2015;373:123-35.

4. Crinò L, Bronte G, Bidoli P, et al. Nivolumab and brain metastases in patients with advanced non-squamous nonsmall cell lung cancer. Lung Cancer 2019;129:35-40.

5. Di Giacomo AM, Valente $M$, Covre A, et al. Immunotherapy targeting immune check-point(s) in brain metastases. Cytokine Growth Factor Rev 2017;36:33-8.

6. Hendriks LEL, Henon C, Auclin E, et al. Outcome of Patients with Non-Small Cell Lung Cancer and Brain Metastases Treated with Checkpoint Inhibitors. J Thorac Oncol 2019;14:1244-54.

7. Bhalla N, Brooker R, Brada M. Combining immunotherapy and radiotherapy in lung cancer. J Thorac Dis 2018;10:S1447-60.

8. Liu Y, Dong Y, Kong L, et al. Abscopal effect of radiotherapy combined with immune checkpoint inhibitors. J Hematol Oncol 2018;11:104.

9. Ahmed KA, Kim S, Arrington J, et al. Outcomes targeting the PD-1/PD-L1 axis in conjunction with stereotactic radiation for patients with non-small cell lung cancer brain metastases. J Neurooncol 2017;133:331-8.
10. Deng L, Liang H, Burnette B, et al. Irradiation and antiPD-L1 treatment synergistically promote antitumor immunity in mice. J Clin Invest 2014;124:687-95.

11. Chiang CL, Chan ACY, Chiu KWH, et al. Combined Stereotactic Body Radiotherapy and Checkpoint Inhibition in Unresectable Hepatocellular Carcinoma: A Potential Synergistic Treatment Strategy. Front Oncol 2019;9:1157.

12. Shepard MJ, Xu Z, Donahue J, et al. Stereotactic radiosurgery with and without checkpoint inhibition for patients with metastatic non-small cell lung cancer to the brain: a matched cohort study. J Neurosurg 2019. [Epub ahead of print]. doi: 10.3171/2019.4.JNS19822.

13. Singh C, Qian JM, Yu JB, et al. Local tumor response and survival outcomes after combined stereotactic radiosurgery and immunotherapy in non-small cell lung cancer with brain metastases. J Neurosurg 2019;132:512-7.

14. Azzam G, Park W, Mellon E, et al. P09 Combined use of radiosurgery with concurrent $\mathrm{PD}-1 / \mathrm{PD}-\mathrm{L} 1$ inhibition for metastatic brain lesions of NSCLC. Int J Radiat Oncol Biol Phys 2018;101:E24.

15. Imber BS, Hellmann MD, Kris MG, et al. 3103 Lesion response and intracranial control of brain metastases from non-small cell lung cancer after stereotactic radiosurgery or hypofractionated radiation therapy combined with checkpoint inhibitors. Int J Radiat Oncol Biol Phys 2017;99:E465-6.

16. Schiappacasse L, Alshehri S, Jumeau R, et al. PO0722 Radiosurgery in association with immunotherapy for NSCLC brain metastases: Feasibility and results. Radiother Oncol 2018;127:S369.

17. Kotecha R, Miller JA, Venur VA, et al. Melanoma brain metastasis: the impact of stereotactic radiosurgery, BRAF mutational status, and targeted and/or immunebased therapies on treatment outcome. J Neurosurg 2018;129:50-9.

18. Ahmed KA, Abuodeh YA, Echevarria MI, et al. Clinical outcomes of melanoma brain metastases treated with stereotactic radiosurgery and anti-PD-1 therapy, antiCTLA-4 therapy, BRAF/MEK inhibitors, BRAF inhibitor, or conventional chemotherapy. Ann Oncol 2016;27:2288-94.

19. Chen L, Douglass J, Kleinberg L, et al. Concurrent Immune Checkpoint Inhibitors and Stereotactic Radiosurgery for Brain Metastases in Non-Small Cell Lung Cancer, Melanoma, and Renal Cell Carcinoma. Int J Radiat Oncol Biol Phys 2018;100:916-25.

20. Gabani P, Fischer-Valuck BW, Johanns TM, et al. Stereotactic radiosurgery and immunotherapy in melanoma brain metastases: Patterns of care and treatment 
outcomes. Radiother Oncol 2018;128:266-73.

21. Stokes WA, Binder DC, Jones BL, et al. Impact of immunotherapy among patients with melanoma brain metastases managed with radiotherapy. J Neuroimmunol 2017;313:118-22.

22. Acharya S, Mahmood M, Mullen D, et al. Distant intracranial failure in melanoma brain metastases treated with stereotactic radiosurgery in the era of immunotherapy and targeted agents. Adv Radiat Oncol 2017;2:572-80.

23. Ahmed KA, Abuodeh YA, Echevarria MI, et al. Clinical outcomes of melanoma brain metastases treated with stereotactic radiosurgery and anti-PD-1 therapy, antiCTLA-4 therapy, BRAF/MEK inhibitors, BRAF inhibitor, or conventional chemotherapy. Ann Oncol 2016;27:2288-94.

24. An Y, Jiang W, Kim B, et al. Stereotactic radiosurgery of early melanoma brain metastases after initiation of anti-CTLA-4 treatment is associated with improved intracranial control. Radiother Oncol 2017;125:80-8.

25. Choong ES, Lo S, Drummond M, et al. Survival of patients with melanoma brain metastasis treated with stereotactic radiosurgery and active systemic drug therapies. Eur J Cancer 2017;75:169-78.

26. Diao K, Bian SX, Routman DM, et al. Stereotactic radiosurgery and ipilimumab for patients with melanoma brain metastases: clinical outcomes and toxicity. J Neurooncol 2018;139:421-9.

27. Gaudy-Marqueste C, Dussouil AS, Carron R, et al. Survival of melanoma patients treated with targeted therapy and immunotherapy after systematic upfront control of brain metastases by radiosurgery. Eur J Cancer 2017;84:44-54.

28. Kaidar-Person O, Zagar TM, Deal A, et al. The incidence of radiation necrosis following stereotactic radiotherapy for melanoma brain metastases: the potential impact of immunotherapy. Anticancer Drugs 2017;28:669-75.

29. Knisely JP, Yu JB, Flanigan J, et al. Radiosurgery for melanoma brain metastases in the ipilimumab era and the possibility of longer survival. J Neurosurg 2012;117:227-33.

30. Lanier CM, Hughes R, Ahmed T, et al. Immunotherapy is associated with improved survival and decreased neurologic death after SRS for brain metastases from lung and melanoma primaries. Neurooncol Pract 2019;6:402-9.

31. Nguyen S, Pearson L, Keller A, et al. Overall survival of melanoma brain metastasis patient cohorts treated with stereotactic radiosurgery with or without immunotherapy. Neuro Oncol 2017;19:vi48.
32. Nguyen S, Pearson L, Keller A, et al. MO_37_2820 Improved Overall and Cause-Specific Survival for Melanoma Brain Metastasis Patients Treated with Stereotactic Radiosurgery and Immunotherapy. Int J Radiat Oncol Biol Phys 2018;102:E352.

33. Rauschenberg R, Bruns J, Brutting J, et al. Impact of radiation, systemic therapy and treatment sequencing on survival of patients with melanoma brain metastases. Eur J Cancer 2019;110:11-20.

34. Silk AW, Bassetti MF, West BT, et al. Ipilimumab and radiation therapy for melanoma brain metastases. Cancer Med 2013;2:899-906.

35. Goel A, Kumar RJ, Linam JM, et al. Impact of anti-PD-1 treatment in patients with metastatic melanoma brain lesions treated with stereotactic radiosurgery. Int J Radiat Oncol Biol Phys 2017;99:E75.

36. Henson A, Mc'Tyre E, Ayala-Peacock DN, et al. Outcomes for Metastatic Melanoma Treated With Stereotactic Radiosurgery In the Era of Targeted Systemic Therapies. Int J Radiat Oncol Biol Phys 2016;96:E135-6.

37. Patel KR, Shoukat S, Oliver DE, et al. Ipilimumab and Stereotactic Radiosurgery Versus Stereotactic Radiosurgery Alone for Newly Diagnosed Melanoma Brain Metastases. Am J Clin Oncol 2017;40:444-50.

38. Diao K, Bian SX, Routman DM, et al. Combination ipilimumab and radiosurgery for brain metastases: tumor, edema, and adverse radiation effects. J Neurosurg 2018;129:1397-406.

39. Yusuf MB, Amsbaugh MJ, Burton E, et al. Peri-SRS Administration of Immune Checkpoint Therapy for Melanoma Metastatic to the Brain: Investigating Efficacy and the Effects of Relative Treatment Timing on Lesion Response. World Neurosurg 2017;100:632-640.e4.

40. Kim JM, Miller JA, Kotecha R, et al. The risk of radiation necrosis following stereotactic radiosurgery with concurrent systemic therapies. J Neurooncol 2017;133:357-68.

41. Glenn C, Hughes R, Lanier C, et al. Incidence of Radiation Necrosis in Brain Metastasis Patients Treated with Stereotactic Radiosurgery and Immunotherapy. Int J Radiat Oncol Biol Phys 2019;103:E50.

42. Petrelli F, De Stefani A, Trevisan F, et al. Combination of radiotherapy and immunotherapy for brain metastases: A systematic review and meta-analysis. Crit Rev Oncol Hematol 2019;144:102830.

43. Cairncross JG, Kim JH, Posner JB. Radiation therapy for brain metastases. Ann Neurol 1980;7:529-41.

44. Shoukat S, Marcus DM, Rizzo M, et al. Outcome with 
stereotactic radiosurgery (SRS) and ipilimumab (Ipi) for malignant melanoma brain metastases (mets). J Clin Oncol 2014;32:abstr 9076.

45. Mathew M, Tam M, Ott PA, et al. Ipilimumab in melanoma with limited brain metastases treated with stereotactic radiosurgery. Melanoma Res 2013;23:191-5.

46. Cohen-Inbar O, Shih HH, Xu Z, et al. The effect of timing of stereotactic radiosurgery treatment of melanoma brain metastases treated with ipilimumab. J Neurosurg 2017;127:1007-14.

47. Gong J, Le TQ, Massarelli E, et al. Radiation therapy and PD-1/PD-L1 blockade: the clinical development of an evolving anticancer combination. J Immunother Cancer 2018;6:46.

48. Melosky B, Juergens R, McLeod D, et al. Immune checkpoint-inhibitors and chemoradiation in stage III unresectable non-small cell lung cancer. Lung Cancer 2019;134:259-67.

49. Theelen WSME, Peulen HMU, Lalezari F, et al. Effect of Pembrolizumab After Stereotactic Body Radiotherapy vs Pembrolizumab Alone on Tumor Response in Patients With Advanced Non-Small Cell Lung Cancer: Results of the PEMBRO-RT Phase 2 Randomized Clinical Trial. JAMA Oncol 2019;5:1276-82.

50. Cinausero M, Laprovitera N, De Maglio G, et al. KRAS and ERBB-family genetic alterations affect response to PD-1 inhibitors in metastatic nonsquamous NSCLC. Ther Adv Med Oncol 2019;11:1758835919885540.

51. Sperduto PW, Jiang W, Brown PD, et al. Estimating Survival in Melanoma Patients With Brain Metastases: An Update of the Graded Prognostic Assessment for

Cite this article as: $\mathrm{He} \mathrm{Q}$, Zhang C, Tang S, Li J, Ren Q. Intracranial radiotherapy with or without immune checkpoint inhibition for brain metastases: a systematic review and metaanalysis. Transl Cancer Res 2020;9(10):5909-5924. doi: 10.21037/ tcr-20-902
Melanoma Using Molecular Markers (MelanomamolGPA). Int J Radiat Oncol Biol Phys 2017;99:812-6.

52. Sperduto PW, Yang TJ, Beal K, et al. Estimating Survival in Patients With Lung Cancer and Brain Metastases: An Update of the Graded Prognostic Assessment for Lung Cancer Using Molecular Markers (Lung-molGPA). JAMA Oncol 2017;3:827-31.

53. Zhuang Y, Yuan BY, Chen GW, et al. Association Between Circulating Lymphocyte Populations and Outcome After Stereotactic Body Radiation Therapy in Patients With Hepatocellular Carcinoma. Front Oncol 2019;9:896.

54. Li YD, Lamano JB, Kaur G, et al. Lymphopenia predicts response to stereotactic radiosurgery in lung cancer patients with brain metastases. J Neurooncol 2019;143:337-47.

55. Cho O, Chun M, Kim SW, et al. Lymphopenia as a Potential Predictor of Ipsilateral Breast Tumor Recurrence in Early Breast Cancer. Anticancer Res 2019;39:4467-74.

56. Dovedi SJ, Adlard AL, Lipowska-Bhalla G, et al. Acquired resistance to fractionated radiotherapy can be overcome by concurrent PD-L1 blockade. Cancer Res 2014;74:5458-68.

57. Lehrer EJ, Peterson J, Brown PD, et al. Treatment of brain metastases with stereotactic radiosurgery and immune checkpoint inhibitors: An international meta-analysis of individual patient data. Radiother Oncol 2019;130:104-12.

58. Lu VM, Goyal A, Rovin RA, et al. Concurrent versus nonconcurrent immune checkpoint inhibition with stereotactic radiosurgery for metastatic brain disease: a systematic review and meta-analysis. J Neurooncol 2019;141:1-12. 


\section{Supplementary}

Table S1 The Newcastle-Ottawa Scale (NOS) to evaluate the studies included

\begin{tabular}{|c|c|c|c|c|c|c|c|c|c|c|}
\hline \multirow{2}{*}{ Study } & \multicolumn{4}{|c|}{ Selection } & \multirow{2}{*}{$\frac{\text { Comparability }}{5}$} & \multicolumn{3}{|c|}{ Outcomes } & \multirow{2}{*}{-NOS score } & \multirow{2}{*}{ Quality } \\
\hline & 1 & 2 & 3 & 4 & & 6 & 7 & 8 & & \\
\hline Acharya 2017 (22) & * & * & * & - & ** & * & * & * & 8 & High \\
\hline Ahmed 2016.1 (23) & * & * & * & - & ** & * & * & * & 8 & High \\
\hline Ahmed 2016.2 (18) & * & * & * & - & ** & * & - & * & 7 & High \\
\hline An 2017 (24) & * & * & * & - & ** & * & * & * & 8 & High \\
\hline Chen 2018 (19) & * & * & * & - & ** & * & * & * & 8 & High \\
\hline Choong 2017 (25) & * & * & * & - & ** & * & * & * & 8 & High \\
\hline Diao 2018.1 (38) & * & * & * & - & ** & * & * & * & 8 & High \\
\hline Diao 2018.2 (26) & * & * & * & - & ** & * & * & * & 8 & High \\
\hline Gabani 2018 (20) & * & * & * & - & ** & * & * & * & 8 & High \\
\hline Gaudy-Marqueste 2017 (27) & * & * & * & - & * & * & * & * & 7 & High \\
\hline Glenn 2019 (41) & * & * & * & - & * & * & - & * & 6 & High \\
\hline Goel 2017 (35) & * & * & * & - & * & * & - & * & 6 & High \\
\hline Henson 2016 (36) & * & * & * & - & * & * & - & * & 6 & High \\
\hline Kaidar-Person 2017 (28) & * & * & * & - & ** & * & * & * & 8 & High \\
\hline Kim 2017 (40) & * & * & * & & ** & * & - & * & 7 & High \\
\hline Knisely 2012 (29) & * & * & * & - & ** & * & * & * & 8 & High \\
\hline Kotecha 2018 (17) & * & * & * & - & ** & * & * & * & 8 & High \\
\hline Lanier 2019 (30) & * & * & * & - & ** & * & - & * & 7 & High \\
\hline Nguyen 2018 (32) & * & * & * & - & * & * & - & * & 6 & High \\
\hline Nguyen 2017 (31) & * & * & * & - & * & * & * & * & 7 & High \\
\hline Patel 2017 (37) & * & * & * & - & ** & * & * & * & 8 & High \\
\hline Rauschenberg 2019 (33) & * & * & * & - & ** & * & * & * & 8 & High \\
\hline Shepard 2019 (12) & * & * & * & - & ** & * & * & * & 8 & High \\
\hline Silk 2013 (34) & * & * & * & - & ** & * & * & * & 8 & High \\
\hline Stokes 2017 (21) & * & * & * & - & ** & * & * & * & 8 & High \\
\hline Yusuf 2017 (39) & * & * & * & - & ** & * & * & * & 8 & High \\
\hline
\end{tabular}

Notes: 1. Representativeness of the exposed cohort; 2. Selection of the non-exposed cohort; 3. Ascertainment of exposure; 4. Demonstration that outcome of interest was not present at start of study; 5. Comparability of cohorts on the basis of the design or analysis; 6 . Assessment of outcome; 7. Was follow-up long enough for outcomes to occur; 8. Adequacy of follow-up of cohorts. 